\title{
Mediación en odontología y Garantías Explícitas en Salud en Chile 2005-2014: las diferencias entre el mundo privado y el público
}

\section{Mediation in dentistry and Explicit Healthcare Guarantees in Chile 2005-2014: differences between the private and the public areas}

\author{
Karla Moscoso-Matus ${ }^{1}$, Marco Cornejo-Ovalle ${ }^{2}$
}

1 Cirujano Dentista, Especialista en Odontología Forense, Unidad Auditoría Interna, Servicio Médico Legal, Santiago de Chile. Consejera Fundación Sonrisas

2 Cirujano Dentista, Especialista en radiología Máxilo Facial, Facultad de Odontología,

Universidad de Chile, Santiago de Chile. Director Fundación Sonrisas

Correspondencia a: Dr. Marco Cornejo Ovalle, Facultad de Odontología de la Universidad de Chile. Sergio Livingstone Pohlhammer \#943, Independencia, Santiago. Teléfono: +562 2978 1712

Trabajo recibido el 24/05/2016. Aprobado para su publicación el 08/02/2017

\section{RESUMEN}

Antecedentes: En Chile desde 2005 se implementó la Ley Auge que establece como derecho de los usuarios, beneficiarios tanto del sistema público o privado de salud, cuatro Garantías Explícitas de Salud denominadas GES, entre las que se incluye la garantía de calidad y dentro de ella se establece la mediación como un procedimiento prejudicial, obligatorio, tendiente a evitar la judicialización de los casos de responsabilidad sanitaria. Este procedimiento contempla dos instancias de mediación según el prestador sea público o privado. Objetivo: describir las mediaciones por atenciones odontológicas en ambos subsistemas. Métodos: estudio trasversal descriptivo sobre las mediaciones efectuadas desde la implementación de este procedimiento (año 2005) hasta el año 2014, por prestaciones odontológicas, otorgadas tanto por prestadores públicos como privados. Resultados: En el periodo de estudio, se efectuaron 519 mediaciones en el ámbito odontológico. La mayoría $(60,4 \%)$ de las reclamantes en mediaciones públicas son mujeres. Las especialidades o prestaciones odontológicas más comprometidas, información no disponible para las mediaciones públicas, son por atenciones de endodoncia $(25 \%)$, implantología (16\%) y prótesis $(15 \%)$. El $58 \%$ de las mediaciones privadas y sólo el $34 \%$ de las públicas finalizan con acuerdo total entre las partes, existiendo dificultades para comparar ambos subsistemas, por la amplia variación en la recolección de datos. Entre las mediaciones públicas, un alto porcentaje de los reclamantes $(70 \%)$ solo solicita disculpas y/o atención de salud y no busca retribuciones económicas. Conclusión: las reclamantes en las mediaciones en odontología son mayoritariamente mujeres, las especialidades odontológicas más comprometidas son endodoncia, implantes y prótesis, y la mayoría solicita sólo disculpas/ explicaciones de los hechos y prestaciones asistenciales. Los registros de las mediaciones públicas y privadas contienen información disímil y parcial, por lo que se no se puede concluir sobre sus diferencias.

PALABRAS CLAVES:

mediación, odontología, responsabilidad sanitaria, derechos en salud

Rev. Clin. Periodoncia Implantol. Rehabil. Oral Vol. 10(1); 49-53, 2017.

\section{ABSTRACT}

Background: In Chile since 2005 the AUGE Act establishes the rights of beneficiaries of both the public and private health systems four Explicit Health Guarantees called GES, among them quality assurance. The later included mediation implemented and established as a preliminary procedure, mandatory, intended to avoid prosecution of cases of medical malpractice. This procedure involves two instances of mediation depending on public or private providers. Objective: To describe the mediations arising from dental care in Chile. Methods: A descriptive cross-sectional study on mediatons since the implementation of this procedure (2005) until 2014, by dental benefits granted by both public and private providers. Results: During the study period, 519 mediations were performed in the dental field. The majority $(60.4 \%)$ of complaints in public context are women. Specialties or more committed dental services, data available only for private mediations, are endodontics (25\%), implantology (16\%) and prosthetics (15\%). $58 \%$ of private and only $34 \%$ of public mediatons end up with full agreement between the parties, with difficulties to compare the two systems due to the wide variation in data collection. Among public averages, a high percentage of claimants $(70 \%)$ only request explanations/apologize and/or health benefits and do not economic compensation. Conclusion: claimants are mostly women, the most compromised dental specialties are endodontics, implants and prostheses, and most patients request only apologies/explanations about the facts and provided dental healthcare. The public and private mediations are not entirely comparable since data sources are dissimilar and contain partial information, so it is not possible to conclude about their differences. KEYWORDS:

mediation, dentistry, medical malpractice, health rights

Rev. Clin. Periodoncia Implantol. Rehabil. Oral Vol. 10(1); 49-53, 2017. 


\section{INTRODUCCIÓN}

El año 2005, Chile da inicio a la Reforma de Salud con la puesta en marcha de la Ley Auge ${ }^{1}$ estableciendo como derecho de los usuarios, independiente si son beneficiarios del sistema público o privado de salud, cuatro Garantías Explícitas de Salud denominadas GES para un conjunto de enfermedades. Estas garantías corresponden a protección financiera, oportunidad, acceso y calidad de las prestaciones efectuadas. Para dar cumplimiento a esa nueva legislación y específicamente la garantía de calidad, se instauró un sistema de acreditación institucional y de certificación de los prestadores individuales de salud. Pero además, se estableció un procedimiento pre judicial para aquellos casos en que los usuarios del sistema de salud no estuviesen satisfechos con la atención recibida y decidieran recurrir a la justicia en busca de reparación. Este procedimiento, cuyo objetivo es propiciar que las partes en conflicto lleguen a un acuerdo previo a la judicialización del caso, se denomina mediación. Entre sus características se incluye que se efectúa en el Consejo de Defensa del Estado (CDE) en los casos originados en el sistema público y en la Superintendencia de Salud (SIS), para casos del sistema privado. En ambos sistemas, posee carácter obligatorio en la justicia civil pero no para la justicia penal; se rige por los principios de igualdad de los participantes, confidencialidad, imparcialidad, voluntariedad, probidad; no requiere de representación por parte de un abogado y su duración máxima es de 60 días corridos, ampliable a 120 sólo si ambas partes están de acuerdo. En el caso de las mediaciones efectuadas en el sistema público, de existir acuerdos económicos superiores a 1000 UF (aproximadamente U\$38.000) se requiere la aprobación de Consejo de Defensa del Estado y sobre 3000 UF (aproximadamente U\$ 115.000) del Ministerio de Hacienda. Estas restricciones no aplican al sistema privado.

La literatura nacional muestra los efectos "desjudicializadores",3,4 que este procedimiento ha significado, es decir, se observa una disminución de los casos de responsabilidad sanitaria que en términos efectivos llegan a los tribunales de justicia. También se ha reportado que la tasa de éxito medio de los procedimientos de mediación en salud, esto es el porcentaje de casos resueltos con acuerdo en relación con el total de casos ingresados a tramitación es baja y no supera el $18 \%{ }^{4}$. Sin embargo, existe escasa información que compare la efectividad de la mediación del sistema público y el privado. Más aún, son escasos los estudios en áreas específicas, como la odontología. Por tanto, el objetivo de esta investigación es caracterizar las mediaciones efectuadas en el ámbito de las prestaciones odontológicas otorgadas entre los años 2005 al 2014 tanto del sistema público como el privado.

\section{MATERIAL Y MÉTODO}

Se trata de un estudio transversal y descriptivo sobre las mediaciones efectuadas desde la implementación de este procedimiento (año 2005) hasta el año 2014, por prestaciones odontológicas, otorgadas tanto por prestadores públicos como privados.

Para obtener la información, acogiéndose a La Ley de Transparencia o $N^{\circ} 20.285$ sobre Acceso a la Información Pública (disponible en http://www.portaltransparencia.cl), se solicitó al CDE y a la SIS acceder a los datos de mediaciones efectuadas sobre prestaciones odontológicas otorgadas por el sistema público y privado de salud respectivamente, desde el inicio de la Reforma hasta el año 2014. La información recibida contenía los datos de mediaciones públicas, entre años 2007 y 2013, y entre el año 2005 hasta abril de 2014 para las mediaciones privadas. Los antecedentes aportados fueron complementados con datos secundarios que se encuentran disponibles on line en sus respectivas web institucionales.

Las variables analizadas fueron: sexo del demandante, región de ocurrencia del suceso, año de la mediación, especialidad o prestación odontológica causal de la mediación, prestador reclamado (persona jurídica/natural), vía de finalización de la mediación, resultado de la mediación (con acuerdo/sin acuerdo), tipo de reparación: disculpas, prestaciones asistenciales, indemnización.

De los datos proporcionados por la SIS fueron eliminados 2 registros por ser considerados inconsistentes. Adicionalmente y con el objeto de estandarizar las variables, se procedió a su homologación a partir de los registros obtenidos (Anexo $N^{\circ}$ 1: Homologación de variables)

El análisis consideró la descripción de las variables de estudio mediante frecuencias absolutas y relativas. Además se realizaron pruebas estadísticas de diferencias de proporciones mediante el test de chi cuadrado de Pearson. Los datos fueron analizados utilizando el programa Excel 2013 (Microsoft Office, NY, USA).

\section{RESULTADOS}

Los registros indican que desde el año 2005 a la fecha, se han efectuado 519 mediaciones en el ámbito odontológico. La distribución por sexo de los casos de mediación del sistema público, indican que entre los reclamantes existe un predominio de las mujeres $(60,4 \%)$. La SIS no consigna el sexo del reclamante/reclamado.

En la Tabla 1 se detalla la distribución geográfica del total mediaciones odontológicas por región del país y sistema donde fue efectuada. En el sistema privado, según tipo de prestador reclamado, el $52 \%$ de los casos registrados compromete personas jurídicas.

La distribución total de los casos de mediación odontológica según año, en el sistema privado y público, se muestra en el Gráfico 1. Al respecto, con anterioridad al año 2010 el CDE no separa sus registros estadísticos por especialidad o área. Recién el año 2010 los registros permiten obtener desagregadamente las mediaciones por área, y por tanto estimar cuántas corresponden al ámbito odontológico.

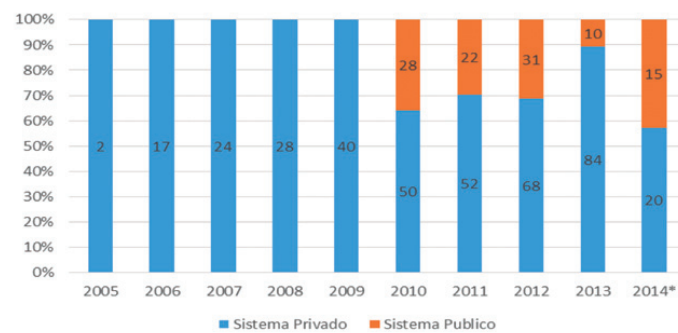

Nota: *Los casos del sistema privado incluyen sólo hasta abril del año 2014.

Grafico 1. Número de mediaciones en el área odontológica efectuadas en el sistema privado y en el sistema público, según año 2010-2014

Respecto a la especialidad o prestación odontológica comprometida, los registros proporcionados por el CDE no especifican si el prestador reclamado es una persona jurídica o natural, ni tampoco la especialidad/prestación odontológica comprometida. De las mediaciones privadas, el mayor porcentaje derivan de atenciones relacionadas con la especialidad de endodoncia, implantología y prótesis (Gráfico 2).

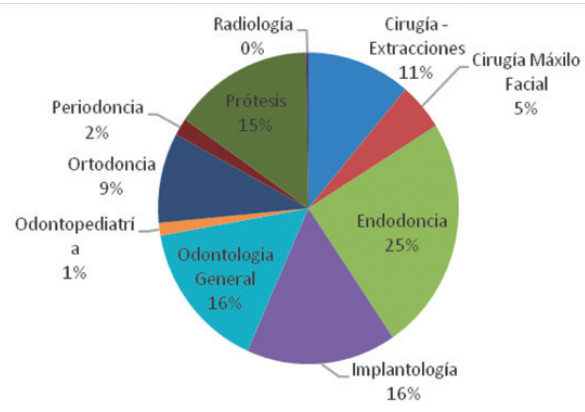

Grafico 2. Distribución porcentual de las mediaciones privadas según especialidades o prestaciones odontológicas. Años 2005-2014

Respecto a las vías en cómo se resuelve una mediación y su distribución según tipo de prestador, se observa en el Gráfico 3. De

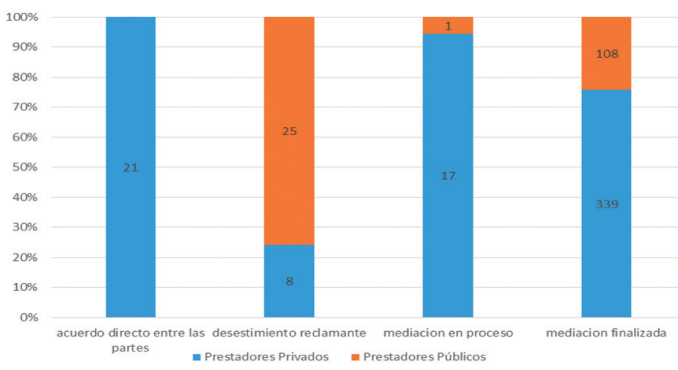

Gráfico 3. Distribución del total de mediaciones, públicas y privadas, según tipo de finalización del proceso de mediación. 
Tabla 1. Distribución del total de casos de mediación odontológica, en el sistema privado de salud (años 2005-2014) y en el sistema público (años 20072013), según región de ocurrencia y tipo de prestador reclamado.

\begin{tabular}{|c|c|c|c|c|c|}
\hline \multirow{3}{*}{ Región de ocurrencia } & \multicolumn{3}{|c|}{$\begin{array}{l}\text { Mediaciones generadas desde el sistema privado de salud. } \\
\qquad \text { Años 2005-2014 }\end{array}$} & \multicolumn{2}{|c|}{$\begin{array}{l}\text { Mediaciones generadas desde el sistema } \\
\text { público de salud. } \\
\text { Años 2007-2013 }\end{array}$} \\
\hline & Tipo de pres & r reclamado & & & \\
\hline & persona jurídica & persona natural & pirvado (70) & punico & pundice (\%) \\
\hline XV Región y I Región & 4 & 9 & $3,38 \%$ & 7 & $5,22 \%$ \\
\hline II Región & 7 & 13 & $5,19 \%$ & 4 & $2,99 \%$ \\
\hline III Región & 3 & 8 & $2,86 \%$ & 5 & $3,73 \%$ \\
\hline IV Región & 10 & 11 & $5,45 \%$ & 6 & $4,48 \%$ \\
\hline Metropolitana & 128 & 75 & $52,73 \%$ & 51 & $38,06 \%$ \\
\hline V Región & 12 & 11 & $5,97 \%$ & 11 & $8,21 \%$ \\
\hline VI Región & 4 & 8 & $3,12 \%$ & 11 & $8,21 \%$ \\
\hline VII Región & 5 & 5 & $2,60 \%$ & 2 & $1,49 \%$ \\
\hline VIII Región & 15 & 21 & $9,35 \%$ & 17 & $12,69 \%$ \\
\hline IX Región & 1 & 8 & $2,34 \%$ & 6 & $4,48 \%$ \\
\hline X y XIV Región & 10 & 12 & $5,72 \%$ & 12 & $8,96 \%$ \\
\hline XI Región & 1 & 1 & $0,52 \%$ & 1 & $0,75 \%$ \\
\hline XII Región & 1 & 2 & $0,78 \%$ & 1 & $0,75 \%$ \\
\hline Total general & 201 & 184 & $100 \%$ & 134 & $100 \%$ \\
\hline
\end{tabular}

las mediaciones efectuadas durante el periodo estudiado, el 3,4\% estaban aún en proceso de resolución (1 del sistema público y 17 del sistema privado). Un bajo número de casos $(4,2 \%)$ logra acuerdo directo entre las partes, previo a la ejecución de la mediación misma, y todas ellas corresponden a mediaciones originadas en el sistema privado.

De las mediaciones en el sistema público, 133 se encuentran efectivamente finalizadas. En el $23,1 \%$ de ellas la causa de finalización es por desistimiento del reclamante. Sin embargo, de las 368 mediaciones privadas finalizadas, sólo $2,2 \%$ fue por la misma causa.

El Gráfico 4 muestra la distribución de las mediaciones finalizadas, excluyendo las resueltas por causales administrativas, según tipo de finalización: con y sin acuerdo, y cuya distribución se detalla según sistema de salud.

Total mediaciones finalizadas, públicas y privadas, sin acuerdo y con acuerdo total

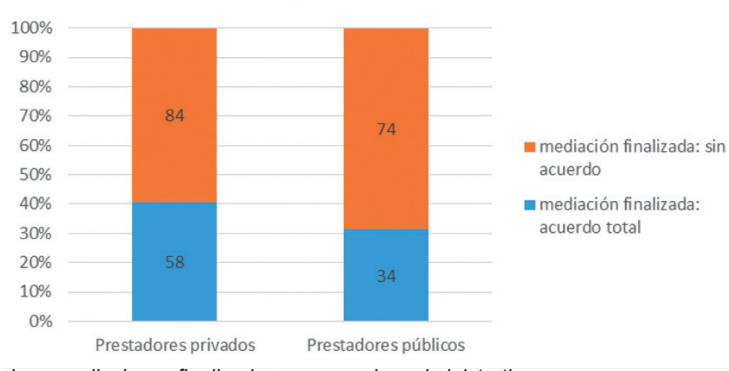

Nota: excluye mediaciones finalizadas por causales administrativas.

Grafico 4. Distribución del total de mediaciones finalizadas, públicas y privadas, según tipo de acuerdo.
Del total de mediaciones finalizadas (250), el 36,8\% logra acuerdo total. No existen diferencias estadísticamente significativas entre ambos subsistemas $(p=0,146)$.

En la Tabla 2 se presenta la distribución de las mediaciones odontológicas realizadas en el sistema público de salud finalizadas con acuerdo total, según tipo de reparación. Dicha información no estuvo disponible en los registros proporcionados por la SIS.

Tabla 2. Tipo de reparación alcanzada en las mediaciones públicas odontológicas finalizadas. Años 2010-2014.

\begin{tabular}{|c|c|c|}
\hline \multirow[t]{2}{*}{ Tipo de reparación } & \multicolumn{2}{|c|}{$\begin{array}{l}\text { Mediaciones finalizadas con } \\
\text { acuerdo total }\end{array}$} \\
\hline & $\mathrm{N}^{\circ}$ & $\%$ \\
\hline $\begin{array}{l}\text { Disculpas y/o explicación de los } \\
\text { hechos }\end{array}$ & 4 & $11,8 \%$ \\
\hline Prestaciones asistenciales & 13 & $38,2 \%$ \\
\hline $\begin{array}{l}\text { Prestaciones asistenciales + disculpas } \\
\text { y/o explicación de los hechos }\end{array}$ & 7 & $20,6 \%$ \\
\hline Indemnización & 4 & $11,8 \%$ \\
\hline $\begin{array}{l}\text { Indemnización + prestaciones } \\
\text { asistenciales }\end{array}$ & 5 & $14,7 \%$ \\
\hline $\begin{array}{l}\text { Indemnización + prestaciones } \\
\text { asistenciales + disculpas y/o } \\
\text { explicación de los hechos }\end{array}$ & 1 & $2,9 \%$ \\
\hline TOTAL & 34 & $100 \%$ \\
\hline
\end{tabular}




\section{DISCUSIÓN}

Dado que Chile posee un componente mixto del aseguramiento en salud, público y privado, el proceso de mediación establecido en la Ley No 19.966 sobre Garantías Explicitas en Salud (GES) debió contemplar dos mecanismos distintos e independientes para llevarlo a cabo. El público a través del CDE y el privado vía la SIS ${ }^{5}$.

Del total de mediaciones generadas en el sistema público desde el año 2010 al 2014, el 1,9\% son de causa odontológica ${ }^{6}$. En el área privada, desde el 2005 a la fecha, han ingresado un total de 4.433 mediaciones admisibles, de ellas, el $7,6 \%$ son de causa odontológica $(0,68 \%$ cirugía cabeza - cuello y maxilofacial y $6,92 \%$ odontología propiamente tal) ${ }^{1}$. Estas mediaciones privadas, representan el $74 \%$ del total de las 519 mediaciones odontológicas generadas en el país. Esté predominio de mediaciones privadas difiere de lo reportado por $\mathrm{Candia}^{4}$ en un estudio sobre mediaciones en salud (aunque no específicamente odontológicas), quien observó mayor proporción de mediaciones públicas. En opinión de estos autores, el reducido número de mediaciones odontológicas públicas no necesariamente se relaciona con que los odontólogos públicos otorguen atenciones de mejor calidad, sino con una cobertura pública de las atenciones odontológicas que apenas supera el $8 \%$ de la población ${ }^{6}$

Respecto a las especialidades más frecuentemente comprometidas se observó que son la endodoncia (25\%), seguido por la implantología y la odontología general, ambas con 16\%. Estos antecedentes concuerdan parcialmente con Portela ${ }^{7}$, quien determinó entre las mediaciones odontológicas privadas, que las especialidades más demandadas eran la endodoncia $(17,95 \%)$, seguida por la prótesis fija $(13,5 \%)$ y exodoncia e implantes, ambas con un $10,9 \%$.

En relación a la naturaleza jurídica del demandado, la SIS consigna $52 \%$ de casos contra personas jurídicas y $48 \%$ contra personas naturales. Estos datos no se correlacionan con el estudio de Portela ${ }^{(7)}$, quien para el mismo sistema encontró una tercera categoría correspondiente a la demanda contra el binomio instituciónprofesional tratante ( $26 \%$ de los casos), además de la categoría "sólo al odontólogo" (29\%) y $22 \%$ "sólo a la institución". Es necesario mencionar, que no es posible efectuar esta diferenciación en las mediaciones públicas, pues según una interpretación de la legislación vigente el prestador público corresponde solo a persona jurídicas ${ }^{1}$ Sin embargo, a nuestro juicio y considerando el contexto actual, donde un porcentaje importante de las atenciones de los programas odontológicos públicos son licitados o contratados a privados, resulta relevante conocer la naturaleza jurídica del prestador

Por otra parte, resulta interesante la diferencia existente entre las mediciones públicas y privadas finalizadas por desistimiento del reclamante $(23,1 \%$ públicas $\mathrm{v} / \mathrm{s} 2,2 \%$ privadas $)$. Al respecto, creemos que estas diferencias podrían explicarse por una tramitación más expedita y/o una mayor experticia de los mediadores del sistema privado $\mathrm{v} / \mathrm{s}$ los del público, favoreciendo la permanencia del reclamante en el proceso. Además, la inexistencia de copago en el sistema público hace plausible que, en ausencia de detrimento económico, los afectados no se vean estimulados a finalizar el trámite. Una tercera razón para esta diferencia podría explicarse en la naturaleza jurídica del demandado, pues la mediación pública será siempre contra una persona jurídica, situación que confiere al pleito la idea de "David contra Goliat", con e desaliento que esto pudiese significar para la persona demandante que son habitualmente los socioeconómicamente menos favorecidos.

Respecto del resultado final de una mediación, este puede ser con o sin acuerdo $2,8,9$. Sin embargo, se debe considerar que existen razones administrativas para dar por terminada una mediación, tales como: inasistencia, expiración de plazos, etc. En razón a ello, podemos decir que del total de mediaciones odontológicas ingresadas, el 96,6\% está finalizado. El $63,2 \%$ del total de mediaciones odontológicas no logra acuerdo, no observándose diferencias estadísticamente significativas entre mediaciones públicas $(6,78 \%)$ y privadas $(11,57 \%)$ que efectivamente finalizan con acuerdo $(p=0,146)$. Los porcentajes de mediaciones odontológicas exitosas son menores que lo observado por Candia ${ }^{4}$ quien, en una revisión de 4.709 mediaciones por atenciones derivadas de todas las áreas de la salud efectuadas entre el 2005 2009 , encontró una tasa de éxito de $15,92 \%$ para las mediaciones públicas y $10,63 \%$ en las privadas y supuso que existe una mayor predisposición de los usuarios del sistema público a encontrar salida al conflicto, de ahí que la tasa de éxito de ese sector sea más alta. A nuestro juicio, una explicación para las diferencias observadas entre ambos estudios podría encontrarse en variaciones metodológicas del cálculo del éxito de la mediación.

Pese a lo magro de las cifras de éxito, según estudios publicados, solo $10,7 \%$ del total de casos de mediciones no exitosas en el sistema público finalizan en un juicio, esto significaría que la mediación tendría un verdadero efecto desjudicializador ${ }^{2,3,4}$. En virtud de ello, sería de interés determinar si se observa el mismo efecto desjudicializador para todas las especialidades y si también está presente en el sistema privado.

Por otra parte, resulta interesante el detalle que efectúa el CDE de los resultados obtenidos tras las mediaciones odontológicas finalizadas, pues consiga que el $11,8 \%$ de los involucrados solo requiere disculpas contra $29,4 \%$ que requiere explicaciones e indemnización, con o sin prestaciones sanitarias asociadas. Estos resultados están alineados con lo publicado a nivel nacional por Lagos $^{3}$, quien determinó que entre las mediaciones en salud en general, un 19\% de los reclamantes solo pide explicaciones. Algo similar se observa a nivel internacional ${ }^{8,9}$, sin embargo, Portela ${ }^{7}$, en un estudio de mediaciones odontológicas nacionales privadas, explícita que el $35 \%$ de los casos solicita indemnización, independiente si además poseen otras pretensiones (por ejemplo, devolución del dinero o nuevo trabajo profesional, con un $23 \%$ y $8 \%$ respectivamente). Paralelamente, el mismo autor hace mención a la existencia de reclamantes que no solicitan ningún tipo de retribución, pero no los cuantifica. Ante esa evidencia, y concordando con estos autores, consideramos que existen dos aspectos que resultarían de suma utilidad ante acciones mévdico legales: primero, los profesionales e instituciones deben acceder a contactarse con los pacientes en busca de un consenso, pues la flexibilidad de las soluciones de la mediación, que incluyen las disculpas, prestaciones asistenciales e indemnizaciones, son un factor predisponente a lograr acuerdos ${ }^{8,9}$. En segundo término, según Riley et $a^{10}$, el juicio de los pacientes hacia las habilidades y calidad de los dentistas está basado en las interacciones personales paciente-odontólogo, el nivel de confort y la sensibilidad post tratamiento. Por tanto, consideramos que sería de gran utilidad social instruir a los alumnos de pregrado o capacitar a los ya egresados, en el establecimiento y profundización de rapport entre profesionales y pacientes, potenciando una buena relación dentista-paciente, así como en los aspectos éticos de la atención en salud ${ }^{11}$. De esta manera, creemos que estas acciones operarían como agentes preventivos de causas judiciales derivadas de la práctica clínica odontológica.

Entre las limitaciones de este estudio se pueden señalar aquellas propias de la calidad de las bases de datos disponibles. Por ejemplo, en las mediaciones privadas no se registran los montos involucrados en las indemnizaciones. Esto resulta relevante pues devela una desigualdad en salud ${ }^{12}$, la cual no sería evidenciable ante la ausencia de la información. Lo anterior se entiende si consideramos que las compensaciones económicas del sistema público están cauteladas a través de montos máximos que requieren la aprobación del CDE y del Ministerio de Hacienda ${ }^{5}$, limitación que no ocurre entre los privados, dando cabida a que estos accedan a compensaciones económicas mucho más altas y/o de manera más fluida. Además, las bases de datos no poseen características sociodemográficas relevantes de los reclamantes, ya que la información entregada por SIS no consignaba antecedentes sociodemográficos del reclamante ni reclamado, como tampoco precisan la definición de "acuerdo parcial".

Como se mencionó anteriormente, se puede evidenciar que el porcentaje de mediaciones públicas odontológicas es bajo, lo que puede explicarse también por la baja cobertura de salud oral ${ }^{6,12}$ Aunque también es importante considerar que en ello pudiesen influir las desigualdades en la alfabetización en salud y el conocimiento de los derechos en salud. Aun siendo ésta la explicación, es importante recalcar el aporte que el proceso de mediación tendría en el empoderamiento de los pacientes sobre sus derechos en salud ${ }^{7}$.

Por otra parte, una fortaleza de esta investigación es que, ante la inexistencia de información consolidada de mediaciones público y privado, este estudio presenta datos de ambos subsistemas, a diferencia de Portela ${ }^{7}$ que sólo considera las ingresadas en el sistema privado.

Finalmente, parece importante discutir las implicancias de la mediación en el contexto actual del ejercicio profesional y del modelo de aseguramiento en salud que garantiza derechos de acceso, oportunidad de atención, protección financiera y calidad de la atención para un grupo importante de problemas de salud ${ }^{12}$, entre los cuales hay 4 del ámbito odontológico ${ }^{1}$. En este marco, nos parece relevante mejorar la abogacía y el empoderamiento en el uso de esta herramienta alternativa para la resolución de conflictos derivados de la atención de salud, como parte de los derechos en salud. Ello facilitaría la participación activa de la población sujeto de esos derechos ante situaciones que afecten los mismos.

Así también, cabe mencionar que aun existiendo un único procedimiento de mediación para resolver los conflictos en salud ${ }^{2}$, su ejecución por parte de dos instituciones diferentes genera una 
amplia disparidad en la recolección de antecedentes, su registro y procesamiento, y más importante aún en el tratamiento de los casos. Nuestro estudio explicita que ante idénticas solicitudes, la variabilidad de los antecedentes enviados por ambas instituciones hace imposible caracterizar cada uno de los mecanismos y menos aún comparar ambos subsistemas. Ante ello, se hace imprescindible que un único ente imparcial consolide la información de antecedentes y resultados de ambas modalidades de este procedimiento. Creemos que así se podrá evaluar de forma real la implementación y los resultados de la mediación en el sistema de salud.

\section{CONTRIBUCIÓN DE LOS AUTORES:}

Ambos autores participaron activamente en el diseño recolección y análisis de datos. Como asimismo en la elaboración del texto y revisión crítica de este.

\section{FUENTES DE APOYO FINANCIERO}

Sin fuentes de apoyo financiero.

\section{CONFLICTOS DE INTERESES}

Los autores declaran no tener conflictos de intereses.

\begin{tabular}{|c|c|}
\hline Descripción original de la SIS & $\begin{array}{l}\text { Homologación de variables } \\
\text { para este estudio }\end{array}$ \\
\hline sin especialidad & odontología general \\
\hline operatoria & odontología general \\
\hline odontología & odontología general \\
\hline Abandono del reclamante & desistimiento reclamante \\
\hline Desistimiento del reclamante & desistimiento reclamante \\
\hline Traslado antecedentes a mediador & mediación en proceso \\
\hline En proceso & mediación en proceso \\
\hline Sin categoría & mediación en proceso \\
\hline Genera mediación & mediación en proceso \\
\hline Oficio orientación a mediación & mediación en proceso \\
\hline Pendiente respuesta prestador & mediación en proceso \\
\hline Prepara Notificación prestador & mediación en proceso \\
\hline Acuerdo directo de las partes & acuerdo directo entre las partes \\
\hline Acuerdo parcial & $\begin{array}{l}\text { mediación finalizada: acuerdo } \\
\text { parcial }\end{array}$ \\
\hline Acuerdo total & $\begin{array}{l}\text { mediación finalizada: acuerdo } \\
\text { total }\end{array}$ \\
\hline Ain acuerdo & mediación finalizada: sin acuerdo \\
\hline Archivo & mediación finalizada \\
\hline Certificado & mediación finalizada \\
\hline Respuesta directa & mediación finalizada \\
\hline
\end{tabular}

\section{Bibliografía}

1. Superintendencia de Salud. Ley $N^{\circ} 19.966$ (AUGE). [consultado 24/08/2015]. Disponible en: http://www.supersalud.gob.cl/normativa/571/w3-article-554.html 2. Unidad de Mediación. Consejo de Defensa del Estado. Mediación y Desjudicialización en el Consejo de Defensa del estado (CDE). Noviembre, 2011. [consultado 29/06/2015]. 28 p. Disponible en: http://www.cde.cl/wps/wcm/ connect/88070669-6e24-4095-9e30-dc43fcf98d42/ESTUDIO+DESJUDICIALIZAC $\mathrm{ON}+\mathrm{version}+$ definitiva+nov+2011. pdf?MOD=AJPERES

3. Lagos S. Impacto de la mediación en la judicialización de conflictos jurídicamente relevantes: un análisis a base de la experiencia chilena de medición por daños en la salud. REJ - Revista de Estudios de la Justicia 2013;18:83-110

4. Candia P; Suazo G. Tasa de éxito del Sistema de Mediación Prejudicial por daños en salud en Chile entre los años 2005 y 2009. Int J Odontostomat 2011;5:300-3

5. Varas, J. Mediación en el ámbito sanitario. Rev Obstet Ginecol Hosp Santiago Oriente Dr, Luis Tisnè Brousse 2011;6: 245-7.

6. Moscoso, K. Carta al editor. Odontólogos de la red pública: cobertura v/s número de profesionales. J Oral Res 2014;3:137-8
7. Portela F. Caracterización de los aspectos bioéticos en los reclamos realizados ante la Superintendencia de Salud de Chile en el área odontológica. Acta Bioethica 2013; 19: 143-52

8. Sohn D, Sonny B. Medical Malpractice Reform: The Role of Alternative Dispute Resolution. Clin Orthop Relat Res 2012;470:1370-8.

9. Brown $H$, Simanowitz A. dispute resolution and mediation. Qual Health Care 1995;4:151-8

10. Riley J, Gordan V, Rindal B, Fellows J, Qvist V. Patel S et al. Components of patient satisfaction with a dental restorative. J Am Dent Assoc. 2012;143:1002-10

11. Fodor A, De La Parra G. La Relación Paciente/Odontólogo: Algunas Consideraciones. Rev Dental Chile 2007;99:21-6

12. Cornejo-Ovalle M, Paraje G, Vásquez-Lavín F, Pérez G, Palència L, Borrell C. Changes in Socioeconomic Inequalities in the Use of Dental Care Following Major Healthcare Reform in Chile, 2004-2009. Int J Environ Res Public Health 2015; 12:2823-36 